\title{
Observancia de las guías internacionales de manejo temprano en sepsis grave y choque séptico
}

\author{
Ricardo Andrés Quintero, Carlos Arturo Martínez, Juan Diego Gamba, Isadora Ortiz, Fabián Jaimes \\ Departamento de Medicina Interna, Facultad de Medicina, Universidad de Antioquia; Hospital Universitario \\ San Vicente de Paúl; Unidad de Investigaciones, Hospital Pablo Tobón Uribe, Medellín, Colombia
}

Introducción. La observancia completa del tratamiento temprano dirigido por objetivos, disminuye significativamente la mortalidad a 28 días, mientras que la observancia parcial no tiene ningún beneficio.

Objetivos. Determinar la observancia de las guías internacionales en sepsis grave y choque séptico, mediante el conjunto de medidas (bundle) de reanimación de las primeras seis horas en pacientes mayores de 16 años de edad, en un centro de referencia de alta complejidad.

Materiales y métodos. Se presenta un estudio de cohorte, prospectivo y descriptivo, llevado a cabo durante un período de seis meses, en pacientes con sepsis grave o choque séptico admitidos a los servicios de urgencias, unidades de cuidados intensivos y cuidados especiales. Se midió la observancia del conjunto de medidas mediante ocho intervenciones: 1) medición del lactato sérico, 2) inicio temprano de antibióticos, 3) toma de hemocultivos, 4) infusión de líquidos intravenosos, 5) uso de medicamentos vasopresores, 6) inserción de catéter venoso central, 7) presión venosa central mayor de $8 \mathrm{~mm} \mathrm{Hg}$, y 8) saturación venosa central de oxígeno mayor de $70 \%$.

Resultados. Se tamizaron 723 pacientes; $16 \%(n=116)$ cumplieron los criterios de inclusión, 92,2 $\%(n=107)$ cumplieron los criterios para sepsis grave y $37,9 \%(n=44)$ para choque séptico. El 62,9 $\%(n=73)$ fue subdiagnosticado. La observancia completa de las 8 intervenciones del conjunto de medidas fue de $0 \%$; de 6 a 7, $19 \%(n=22)$; de 3 a 5, 67,2 \% ( $n=78)$, y de 0 a 2, 98,3\% $(n=114)$.

Conclusión. La completa observancia de las guías internacionales de manejo en sepsis grave y choque séptico en las primeras seis horas es nula y solo se cumple de manera parcial.

Palabras clave: guías, sepsis, choque séptico, observancia, tratamiento.

doi: http://dx.doi.org/10.7705/biomedica.v32i3.349

Adherence to international guidelines on early management in severe sepsis and septic shock

Introduction. Complete adherence to early goal-directed therapy has shown a significant reduction in 28-day mortality rate, whereas partial adherence has not shown beneficial effect.

Objectives. The effect of adherence to 6 hour sepsis bundle treatment was evaluated on patients with severe sepsis and septic shock.

Materials and methods. A prospective cohort study was conducted during a six-month period. The patients were limited to those 16 years or older and included admissions to intensive care units, special care units or emergency departments with severe sepsis or septic shock. The adherence to the 6-hour sepsis bundle was evaluated through 8 interventions as follows: (1) serum lactate measure,(2) early antibiotic administration, (3) blood culture samples, (4) infusion of intravenous fluids, (5) vasopressor use, (6) central venous catheter, (7) central venous pressure $>8 \mathrm{~mm} \mathrm{Hg}$, and (8) central venous oxygen saturation $>70 \%$.

Results. Seven hundred and twenty three patients were screened; $16 \%(n=116)$ met the inclusion criteria; $92.2 \%(n=107)$ met the criteria for severe sepsis and 37.9\% (44) for septic shock; $62.9 \%(n=73)$ were subdiagnosed. Complete adherence to the 8 interventions of 6 -hour sepsis bundle was $0 \% ; 6$ to $7(19 \%, n=22), 3$ to $5(67.2 \%, n=78)$, and 0 to $2(98.3 \%, n=114)$.

Conclusion. Adherence to 6-hour sepsis bundle treatment for severe sepsis and septic shock was not done for the full 8 interventions and only partial accomplishment of the first 4 interventions.

Key words: Guidelines, sepsis, septic shock, adherence, treatment.

doi: http://dx.doi.org/10.7705/biomedica.v32i3.349

\section{Contribución de los autores:}

Ricardo Andrés Quintero, Carlos Arturo Martínez, Juan Diego Gamba e Isadora Ortiz: participaron en el diseño del estudio, la recolección de datos, el análisis de la información y la escritura del manuscrito.

Fabian Jaimes participó en el diseño del estudio, el análisis de la información y la revisión del manuscrito. 
La sepsis es una de las enfermedades más prevalentes y una de las principales causas de muerte en los pacientes hospitalizados (1). La sepsis grave y el choque séptico son dos grandes problemas de salud que afectan a millones de personas alrededor del mundo cada año, y que han presentado un significativo aumento en su frecuencia en los últimos 20 años (1). Se han hecho grandes esfuerzos para desarrollar medidas de alto impacto en su diagnóstico y manejo; es así como en las últimas dos décadas, la definición de sepsis ha evolucionado mediante el debate constante, hasta conformar los criterios diagnósticos actuales de la conferencia de consenso del 2001 (2). Con estos criterios se pretende una definición más apropiada que permita implementar medidas dirigidas al tratamiento temprano y efectivo, en busca de disminuir la morbimortalidad relacionada con la sepsis. En este sentido, se ha desarrollado la Surviving Sepsis Campaign, propuesta desde el año 2002 para promover la aplicación oportuna de una serie de medidas orientadas al diagnóstico y el tratamiento de la sepsis grave y el choque séptico (3).

A pesar de la información disponible y de las recomendaciones de expertos, existe preocupación porque la aplicación de las guías actuales se hace de una manera variable e inconstante, lo cual afecta su impacto potencial sobre la morbilidad y mortalidad. Por lo tanto, se hace prioritaria la aplicación de estrategias de evaluación y educación médica en estos aspectos, con el fin de mejorar la observancia de las recomendaciones internacionales. Además, se ha demostrado que la aplicación juiciosa de dichos procesos de atención mejora significativamente la mortalidad intrahospitalaria (4).

La implementación de las guías de la Surviving Sepsis Campaign se ha hecho mediante la definición de un conjunto de medidas (bundle). En términos de práctica médica, estas se han definido como un conjunto de intervenciones dirigido hacia una enfermedad en particular que, cuando se aplican juntas, producen mejores resultados que cuando se aplican por separado

\section{Correspondencia:}

Ricardo Andrés Quintero, Calle $31 \mathrm{~N}^{\circ} 13 \mathrm{~A}-51$, Edificio Panorama, apartamento 1507, torre 2, Bogotá, D.C., Colombia

Teléfonos: (571) 6965143 y (310) 4076233

rquinterof@gmail.com

Recibido: 06/05/11; aceptado:15/05/12
(5). El conjunto de medidas de las primeras seis horas, llamado el de reanimación, está enfocado en la identificación de pacientes con sepsis grave o choque séptico, el tratamiento temprano dirigido por objetivos, la toma oportuna de muestras para cultivos y el inicio de antibióticos en la primera hora. Varias investigaciones alrededor del mundo (4, 6-10) han evaluado la observancia, percepción y asimilación del personal médico a dichas guías, en el entorno de los servicios hospitalarios de diferente complejidad. No existe en nuestro país una aproximación similar al problema, a pesar del conocimiento actual de la carga de morbilidad y mortalidad que representa la sepsis (11).

El propósito de este estudio fue determinar cómo son tratados los pacientes con sepsis grave y choque séptico en un hospital universitario de alta complejidad, específicamente en cuanto al cumplimento de las guías de tratamiento (conjunto de medidas) recomendadas para las primeras seis horas de atención. Lo anterior, se hace como punto de partida para definir estrategias que generen un proceso dirigido al mejoramiento de la atención y, por ende, al pronóstico de los pacientes.

\section{Materiales y métodos}

\section{Diseño del estudio}

Se trata de un estudio de cohorte, prospectivo y descriptivo, en un centro de referencia de alta complejidad, que se llevó a cabo entre el $1^{\circ}$ de junio y el 30 de noviembre de 2010. Se tamizaron pacientes mayores de 16 años que fueron admitidos consecutivamente en los servicios de urgencias, las unidades de cuidados intensivos y las unidades de cuidados especiales, con diagnóstico inicial de cualquier tipo de infección.

El diagnóstico de infección se hizo con base en las definiciones propuestas por los Centers for Disease Control and Prevention (CDC) de Atlanta (12), incluyendo el diagnóstico de sepsis clínica, que debe cumplir los siguientes tres criterios:
1) hemocultivos negativos o no realizados,
2) sin infección confirmada en otro sitio, y
3) con inicio de tratamiento contra la sepsis (12).

De esta población elegible, se incluyeron de manera definitiva aquellos pacientes que cumplían los criterios de sepsis grave o choque séptico, de acuerdo con los registros de la historia clínica de los médicos tratantes, quienes desconocían el propósito y la conducción de la investigación. 
Los criterios de sepsis grave o choque séptico que debían estar registrados en la historia clínica eran cualquiera de estos:

1) presión sistólica menor de $90 \mathrm{~mm} \mathrm{Hg}$ o presión arterial media menor de $65 \mathrm{~mm} \mathrm{Hg}$, a pesar de la reanimación con líquidos, o disminución de la presión sistólica por debajo de $40 \mathrm{~mm} \mathrm{Hg}$ con respecto a la presión basal;

2) infiltrados pulmonares bilaterales con necesidad de $\mathrm{O}_{2}$ para mantener la saturación de $\mathrm{O}_{2}$ por encima de $90 \%$;

3) hipoxemia $\left(\mathrm{PaO}_{2} / \mathrm{FIO}_{2}\right.$ menor de 300);

4) oliguria (gasto urinario de menos de $0,5 \mathrm{ml} / \mathrm{kg}$ por hora durante, al menos, dos horas);

5) creatinina sérica mayor de $2 \mathrm{mg} / \mathrm{dl}$ o aumento en valores previos de más de $0,5 \mathrm{mg} / \mathrm{dl}$;

6) coagulopatía demostrada por un índice estandarizado internacional (International Normalized Ratio, INR) mayor de 1,5, un tiempo parcial de tromboplastina de más de 60 segundos, o un recuento de plaquetas menor

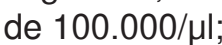

7) bilirrubina sérica total mayor de $2 \mathrm{mg} / \mathrm{dl}$, y

8) lactato sérico por encima de $4 \mathrm{mmol} / \mathrm{L} \circ 36 \mathrm{mg} /$ dl (2).

Se excluyeron los pacientes con diagnóstico de sepsis grave o choque séptico que ingresaran remitidos de otra institución con administración previa de antibióticos; aquellos con alta hospitalaria en las siguientes seis horas del ingreso al hospital; aquellos con cambio en el diagnóstico inicial en las primeras seis horas del ingreso, y las pacientes obstétricas.

Los datos fueron recolectados prospectivamente usando un formato impreso prediseñado para tal fin. Se incluyeron datos demográficos, enfermedades concomitantes, tipo de infección inicial y cumplimiento de criterios diagnósticos de sepsis grave o choque séptico. Los médicos encargados de la investigación y de la recolección de la información, no tuvieron injerencia en las decisiones de diagnóstico, tratamiento y manejo de los pacientes.

La evaluación de la observancia del conjunto de medidas de las primeras seis horas en el manejo de los pacientes, se determinó por medio del cumplimiento de los siguientes elementos en la historia clínica:
1) medición del lactato sérico;

2) administración temprana (una a tres horas) de antibióticos de amplio espectro;

3) toma de muestras para hemocultivos antes del inicio de antibióticos;

4) administración de cristaloides o coloides intravenosos en volúmenes adecuados de acuerdo con los valores recomendados;

5) uso de vasopresores según necesidad de acuerdo con los valores de presión arterial media o presión arterial sistólica;

6) inserción de catéter venoso central;

7) medición de presión venosa central y logro de meta de un valor mayor de $8 \mathrm{~mm} \mathrm{Hg}, \mathrm{y}$

8) medición de saturación venosa central de oxígeno $\left(\mathrm{SvO}_{2}\right)$ y logro de meta de un valor mayor de $70 \%$.

Se hizo seguimiento del paciente hasta el alta de la institución para determinar su estado vital. Los formularios de recolección diligenciados se revisaron semanalmente, al igual que los reportes periódicos de la base de datos, en el centro coordinador de datos para detectar inconsistencias, valores extremos o faltantes, o dificultades en el proceso de reclutamiento.

\section{Análisis estadístico}

Dada la naturaleza puramente descriptiva y exploratoria de la investigación, no se hizo un cálculo formal de tamaño de muestra ni se plantean análisis estadísticos de inferencia. Las variables continuas se presentan como medias y desviación estándar o medianas y rangos intercuartílicos según la distribución de los datos. Las variables nominales se presentan como porcentajes.

\section{Resultados}

En el periodo del estudio, ingresaron al hospital 723 pacientes con sospecha diagnóstica de proceso infeccioso activo (figura 1), entre los cuales predominaron infección urinaria sintomática $(n=164$, $22,7 \%$ ), infección de tejidos blandos ( $n=150,21 \%$ ) y neumonía adquirida en la comunidad ( $n=115$, $16 \%)$ (cuadro 1). De esta población, $16 \%(n=116)$ cumplían los criterios de sepsis grave o choque séptico, y las principales infecciones diagnosticadas fueron neumonía adquirida en la comunidad $(n=29$, $25 \%)$, infección urinaria ( $n=28,24 \%$ y sepsis clínica ( $\mathrm{n}=17,15 \%)$ (cuadro 2). 


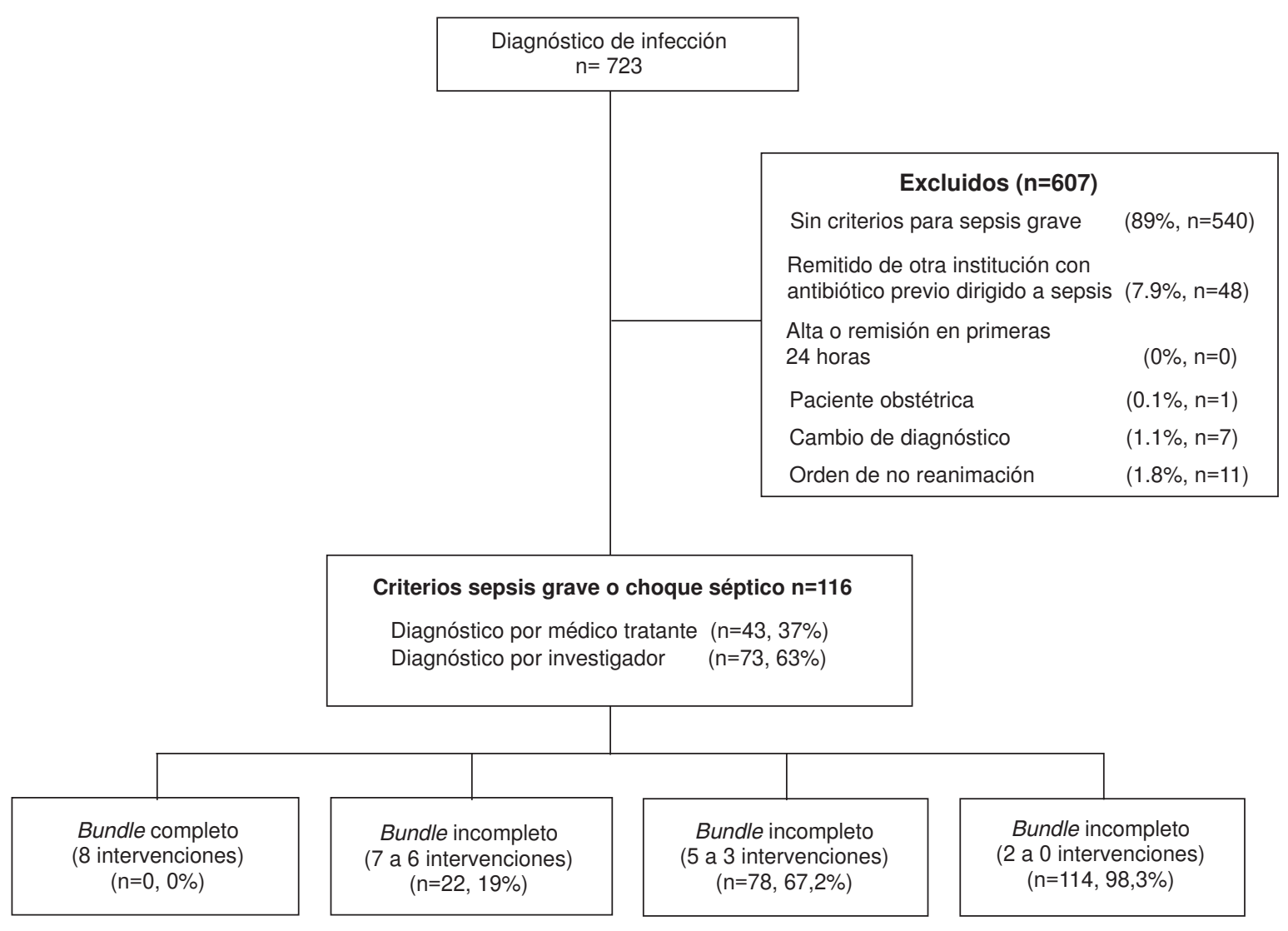

Figura 1. Flujograma de reclutamiento de pacientes con sepsis grave o choque séptico, Hospital Universitario San Vicente de Paúl, Medellín, junio a noviembre de 2010

La mediana de edad fue 61 años [rango intercuartílico (IQR): 47-73]; $53 \%(\mathrm{n}=62)$ eran hombres, la mayoría de raza mestiza ( $n=98,86 \%)$, seguidos por la raza blanca $(n=7,6 \%)$, afro-descendientes $(n=7,6 \%)$ e indígenas $(n=2,2 \%)$. La mayoría de pacientes se detectaron en el servicio de urgencias de la institución (60\%, $n=70)$, seguido por las unidades de cuidados intensivos (27\%, $\mathrm{n}=31)$ y las de cuidados especiales (13\%, $\mathrm{n}=15)$. Las enfermedades concomitantes registradas se describen en orden de frecuencia en el cuadro 3. La mediana de estancia hospitalaria fue de 17 días (IQR: 11-27) y la mortalidad intrahospitalaria fue $19 \%(n=22)$.

Del total de pacientes con diagnóstico de sepsis grave o choque séptico, $37 \% \quad(n=43)$ fueron registrados con dicha condición en la historia clínica por parte del médico tratante, mientras que $63 \%$ ( $n=73)$ cumplían con criterios para sepsis grave o choque séptico documentados en la historia clínica pero no fueron diagnosticados como tal. En ninguno de los 116 pacientes incluidos en la cohorte se aplicó el conjunto completo de medidas de reanimación para las seis primeras horas de atención. De las ocho intervenciones, se cumplieron de 6 a 7 en $19 \%(n=22)$ de 3 a 5 en $67,2 \%(n=78)$, y de 0 a 2 en $98,3 \%(n=114)$ (figura 1$)$.

Al evaluar la observancia en la ejecución de cada uno de los componentes del conjunto de medidas de reanimación para las primeras seis horas, se encontró que el ácido láctico sérico se midió en el $77,6 \%(n=90)$, y el valor promedio fue de $29,7 \mathrm{mg} / \mathrm{dl}(\mathrm{DE}=28,9)$. Se iniciaron antibióticos intravenosos de amplio espectro en $91 \%$ de los casos ( $n=105)$ y, de este grupo, se administró el antibiótico en la primera hora en $14,3 \% \quad(n=15)$, de 1 a 3 horas en $23,8 \%(n=25)$, y luego de las primeras 3 horas en $61 \%(n=64)$. Se tomaron muestras para hemocultivos antes del inicio de los antibióticos en $81,4 \%$ de los casos $(n=79)$.

El $46,1 \%$ de los pacientes $(n=53$ ) presentó criterios de hipoperfusión (lactato sérico mayor de $36 \mathrm{mg} / \mathrm{dl}$ o $4 \mathrm{mmol} / \mathrm{L}$, o hipotensión arterial); de este grupo, el $92,5 \%(n=49)$ se reanimó adecuadamente con 
Cuadro 1. Diagnóstico de ingreso en pacientes admitidos con sospecha de infección, Hospital Universitario San Vicente de Paúl, junio a noviembre de 2010

\begin{tabular}{lrr}
\hline Diagnóstico de ingreso & $\mathbf{n}$ & $\%$ \\
\hline Infección sintomática de vías urinarias & 164 & 22,68 \\
Infección de tejidos blandos & 150 & 20,75 \\
Neumonía adquirida en la comunidad & 115 & 15,91 \\
Infección intraabdominal & 102 & 14,11 \\
Sepsis clínica & 46 & 6,36 \\
Gastroenteritis & 25 & 3,46 \\
Infección del sitio quirúrgico u órgano/espacio & 16 & 2,21 \\
Neumonía hospitalaria & 14 & 1,94 \\
Infección superficial de sitio quirúrgico & 13 & 1,80 \\
Meningitis o ventriculitis & 13 & 1,80 \\
Bacteriemia asociada a catéter & 11 & 1,52 \\
Infección de tejidos blandos & 11 & 1,52 \\
Infección de articulaciones o tejido periarticular & 10 & 1,38 \\
Infección del sistema reproductor & 9 & 1,24 \\
Infección profunda del sitio quirúrgico & 8 & 1,11 \\
Infección de piel & 4 & 0,55 \\
Infección del torrente sanguíneo & 4 & 0,55 \\
Infección intracraneal & 3 & 0,41 \\
Endocarditis & 2 & 0,28 \\
Mediastinitis & 2 & 0,28 \\
Otras infecciones urinarias & 1 & 0,14 \\
Total & 723 & 100 \\
\hline & &
\end{tabular}

Cuadro 2. Diagnósticos de ingreso en pacientes con sepsis grave o choque séptico, Hospital Universitario San Vicente de Paúl, junio a noviembre de 2010

\begin{tabular}{lrr}
\hline Infección principal & n & \multicolumn{1}{c}{$\%$} \\
\hline Neumonía adquirida en la comunidad & 29 & 25,00 \\
Infección sintomática del vías urinarias & 28 & 24,14 \\
Sepsis clínica & 17 & 14,66 \\
Infección intraabdominal & 15 & 12,93 \\
Gastroenteritis & 7 & 6,03 \\
Infección de tejidos blandos & 7 & 6,03 \\
Neumonía hospitalaria & 5 & 4,31 \\
Meningitis o ventriculitis & 2 & 1,72 \\
Bacteriemia asociada a catéter & 1 & 0,86 \\
Endocarditis & 1 & 0,86 \\
Infección de articulaciones o tejido periarticular & 1 & 0,86 \\
Infección de tejidos blandos & 1 & 0,86 \\
Infección de piel & 1 & 0,86 \\
Mediastinitis & 1 & 0,86 \\
Total & 116 & 100,00 \\
\hline
\end{tabular}

líquidos intravenosos $(20 \mathrm{ml} / \mathrm{kg}$ de cristaloides o $1.500 \mathrm{ml}$ en bolo), mientras que $7,6 \%(n=4)$ los recibieron en cantidad insuficiente. En los pacientes hipoperfundidos que recibieron reanimación adecuada con líquidos intravenosos, se lograron metas de presión arterial media de $65 \mathrm{~mm} \mathrm{Hg}$ o más en $38,8 \%(n=19)$ y no se alcanzó este objetivo en $61,2 \%(n=30)$. En este último grupo se iniciaron vasopresores en $96,7 \%(n=29)$, y se recurrió al uso de dopamina en $72,4 \%(n=21)$ y de norepinefrina en $27,6 \%(n=8)$, lográndose metas
Cuadro 3. Enfermedades concomitantes en los pacientes incluidos $^{1}$

\begin{tabular}{lcc}
\hline Enfermedad & $\mathbf{n}$ & $\%$ \\
\hline Enfermedad renal crónica & 31 & 26,72 \\
Diabetes mellitus & 29 & 25,00 \\
Enfermedad pulmonar obstructiva crónica & 25 & 21,55 \\
Uso crónico de esteroides & 13 & 11,21 \\
Falla cardiaca congestiva & 12 & 10,34 \\
Paciente con trasplante & 12 & 10,34 \\
Cáncer en el último año & 7 & 6,03 \\
Drogadicción/alcoholismo & 6 & 5,17 \\
Trauma o cirugía & 5 & 4,31 \\
VIH-sida & 3 & 2,63 \\
Cirrosis hepática & 1 & 0,86 \\
\hline
\end{tabular}

${ }^{1}$ Algunos pacientes pueden presentar más de una enfermedad concomitante.

de presión arterial media de $65 \mathrm{~mm} \mathrm{Hg}$ o más en $89,7 \%(n=26)$.

Se insertó catéter venoso central en $28,4 \%$ de los pacientes $(n=33)$; de este grupo, se logró la meta de presión venosa central de $8 \mathrm{~mm} \mathrm{Hg}$ o más en $42,4 \%(n=14)$. Se midió la saturación venosa central de oxígeno $\left(\mathrm{SvcO}_{2}\right)$ en $5,17 \%(n=6)$, con logro de meta de $\mathrm{SvO}_{2}$ de $70 \%$ o más en $50 \%(n=3)$.

\section{Discusión}

Han pasado más de 10 años desde la publicación del estudio de Rivers, et al., en el cual demostraron el beneficio de una disminución del $16 \%$ en la mortalidad intrahospitalaria con la implementación del tratamiento temprano dirigido por objetivos durante las primeras seis horas (13). A partir de entonces, se han corroborado los beneficios de esta estrategia de reanimación en múltiples estudios $(9,10,14-17)$. A pesar de los hallazgos y de ser una estrategia recomendada en las guías internacionales y por importantes sociedades e instituciones, es preocupante ver la poca observancia a las guías detectada en diferentes sitios del mundo.

Las tasas de observancia del conjunto de medidas de reanimación reportadas en diferentes estudios varían entre 5 y $50 \%$, y se ha demostrado que, en el contexto de un proceso de educación continuada, se puede mejorar la observancia e impactar en la mortalidad de los pacientes (4).

El presente estudio en un hospital de referencia es, a nuestro entender, el primer estudio colombiano que evalúa la observancia del conjunto de medidas de reanimación en pacientes con sepsis grave y choque séptico según las recomendaciones de la Surviving Sepsis Campaing y el Institute for Healthcare Improvement (3). La escasa observancia 
de dichas recomendaciones demuestra la dificultad de trasladar los conocimientos a la práctica $(7,10)$.

Estudios similares al nuestro, desarrollados en otros países, muestran que a la fecha pocas instituciones en el mundo han adoptado un protocolo formal para el tratamiento temprano dirigido por objetivos en el manejo de la sepsis grave y el choque séptico $(6,7)$ y, aun en instituciones con protocolos establecidos, la observancia de las guías varía entre el 50 y el $60 \%(7,10)$. Un estudio reciente demostró que el conjunto de medidas de reanimación no se implementó en $42 \%$ de los pacientes elegibles y que, en aquellos en quienes se inició el protocolo, este se desarrolló de manera incompleta en $43 \%$ de los casos (18).

En la literatura científica se han descrito varias barreras potenciales para la observancia de las guías (18). En este estudio, se pueden sugerir las siguientes. Primero, existen limitaciones en el diagnóstico de la sepsis grave por diversas razones, como la falta de entrenamiento en el reconocimiento de este tipo de pacientes, partiendo de la misma formación en pregrado hasta la ausencia de guías diagnósticas y terapéuticas validadas localmente para los profesionales que trabajan en las instituciones de salud. Lo anterior tiene como consecuencia, entre otras, el no practicar los exámenes necesarios para identificar la disfunción de un órgano como, por ejemplo, la detección de hipoperfusión tisular mediante la medición de lactato sérico. Esta podría ser una de las principales razones para nuestros resultados, sustentada en la discordancia entre los pacientes con diagnóstico de sepsis grave hecho por su médico tratante (37\%), y aquellos con diagnóstico hecho por los investigadores (63\%) con base en los datos de la historia clínica y los exámenes paraclínicos.

En segundo lugar, y de alguna manera consecuencia de la anterior, parece lógico encontrar una baja tasa de aplicación del protocolo de reanimación en aquellos pacientes que cursan con choque oculto (definido como la presencia de concentraciones de lactato sérico mayores de $36 \mathrm{mg} / \mathrm{dl}$ o $4 \mathrm{mmol} / \mathrm{L}$ sin evidencia de hipotensión arterial), pero sin el diagnóstico correcto por no encontrar en el examen físico una disminución de la presión arterial.

Tercero, si bien en algunos pacientes se establece con prontitud el diagnóstico de sepsis grave y se inicia el manejo apropiado en sus aspectos básicos (reanimación con líquidos, imágenes y exámenes básicos de laboratorio, toma de muestras para microbiología, inicio temprano de antibióticos, etc.), en la gran mayoría de los casos no se cumple todo el protocolo del conjunto de medidas de reanimación por la necesidad de practicar procedimientos invasivos que no son de fácil acceso en todos los servicios de hospitalización o urgencias (inserción de catéteres venosos centrales o catéteres arteriales) debido, frecuentemente, a la demanda desbordada de los servicios de salud y a las limitaciones en tiempos de atención relacionadas con la forma de operación del sistema de salud nacional actual; por esta razón, muchas veces no es posible llevar a cabo las mediciones fundamentales para guiar la reanimación, tales como la saturación venosa central de oxígeno.

Es importante desde ahora buscar estrategias educativas dirigidas a adoptar y adaptar las guías internacionales de manera sistemática en todas las instituciones de salud de nuestra nación, mediante la creación de grupos de trabajo académico, reconocidos y avalados no sólo en el ámbito de las universidades sino también por las diferentes sociedades científicas y asociaciones profesionales. Dichasguíasdeberíanimplementarse con miras a hacer parte de la evaluación de los estándares de buena práctica médica.

Debido a la gran preocupación sobre la poca observancia de las guías a nivel mundial, la Surviving Sepsis Campaing ha creado un programa para la mejoría del desempeño (19), una de cuyas partes fundamentales es la creación de materiales educativos y de un registro internacional en donde las instituciones pueden ingresar los datos de sus pacientes y seguir su rendimiento. Los resultados de este programa muestran cómo, en un periodo de dos años, la observancia inicial del conjunto de medidas de reanimación mejoró de 10,5\% a $31,3 \%$, aumento que parece asociarse con una reducción en la mortalidad (20).

En Latinoamérica, países como Brasil han demostrado que el cumplimiento de esta estrategia es posible mediante un trabajo coordinado en hospitales públicos y privados (21). Esta intervención es liderada por el Instituto Latinoamericano de Sepsis (www.sepsisnet.org) y tiene el objetivo de promover herramientas de manejo efectivas contra la sepsis.

En conclusión, este estudio demuestra que en un hospital universitario de alta complejidad en Colombia existe una baja observancia del conjunto 
de medidas de reanimación en pacientes con sepsis grave y choque séptico, lo cual no es ajeno a la realidad mundial. Por su diseño, en este estudio no se puede concluir específicamente sobre cuáles son las causas de esta escasa observancia ni cómo dicho fenómeno puede influir el pronóstico de los pacientes, preguntas que deben ser objeto de otras investigaciones. No obstante, ante la magnitud del problema (11) y los hallazgos en otras latitudes sobre los beneficios de esta intervención $(7,10,22)$, se justifica hacer esfuerzos institucionales y gubernamentales para la implementación adecuada de los protocolos de manejo en sepsis.

\section{Conflicto de intereses}

Los autores manifiestan no tener ningún conflicto de intereses para la realización del presente estudio.

\section{Financiación}

La financiación del proyecto se consiguió mediante recursos propios aportados por los investigadores.

\section{Referencias}

1. Angus DC, Linde-Zwirble WT, Lidicker J, Clermont G, Carcillo J, Pinsky MR. Epidemiology of severe sepsis in the United States: Analysis of incidence, outcome, and associated costs of care. Crit Care Med. 2001;29:1303-10.

2. Levy MM, Fink MP, Marshall JC, Abraham E, Angus D, Cook D, et al. 2001 SCCM/ESICM/ACCP/ATS/SIS International Sepsis Definitions Conference. Crit Care Med. 2003;31:1250-6.http://dx.doi.org/10.1097/01.CCM.000005 0454.01978.3B

3. Dellinger RP, Levy MM, Carlet JM, Bion J, Parker MM, Jaeschke R, et al. Surviving Sepsis Campaign: International guidelines for management of severe sepsis and septic shock: 2008. Crit Care Med. 2008;36:296-327. http://dx.doi. org/10.1097/01.CCM.0000298158.12101.41

4. Ferrer R, Artigas A, Levy MM, Blanco J, González-Díaz G, Garnacho-Montero J, et al. Improvement in process of care and outcome after a multicenter severe sepsis educational program in Spain. JAMA. 2008;299:2294-303. http://dx.doi.org/10.1001/jama.299.19.2294

5. Dellinger RP, Vincent JL. The Surviving Sepsis Campaign sepsis change bundles and clinical practice. Crit Care. 2005;9:653-4. http://dx.doi.org/10.1186/cc3952

6. Brunkhorst FM, Engel C, Ragaller M, Welte T, Rossaint R, Gerlach H, et al. Practice and perception a nationwide survey of therapy habits in sepsis. Crit Care Med. 2008;36:271925. http://dx.doi.org/10.1097/CCM.0b013e318186b6f3

7. Gao F, Melody T, Daniels DF, Giles S, Fox S. The impact of compliance with 6-hour and 24-hour sepsis bundles on hospital mortality in patients with severe sepsis: A prospective observational study. Crit Care. 2005;9:R764-70. http://dx.doi.org/10.1186/cc3909
8. Jones AE, Focht A, Horton JM, Kline JA. Prospective external validation of the clinical effectiveness of an emergency department-based early goal-directed therapy protocol for severe sepsis and septic shock. Chest. 2007;132:425-32. http://dx.doi.org/10.1378/chest.07-0234

9. Trzeciak S, Dellinger RP, Abate NL, Cowan RM, Stauss M, Kilgannon JH. Translating research to clinical practice: A 1-year experience with implementing early goal-directed therapy for septic shock in the emergency department. Chest. 2006;129:225-32. http://dx.doi.org/10.1378/chest.129. 2.225

10. Nguyen HB, Corbett SW, Steele R, Banta J, Clark RT, Hayes SR, et al. Implementation of a bundle of quality indicators for the early management of severe sepsis and septic shock is associated with decreased mortality. Crit Care Med. 2007;35:1105-12. http://dx.doi.org/10.1097/01. CCM.0000259463.33848.3D

11. Rodríguez F, Barrera L, De La Rosa G, Dennis R, Dueñas C, Granados M, et al. The epidemiology of sepsis in Colombia: A prospective multicenter cohort study in ten university hospitals. Crit Care Med. 2011;39:1675-82. http:// dx.doi.org/10.1097/CCM.0b013e318218a35e

12. Garner JS, Jarvis WR, Emori TG, Horan TC, Hughes JM. CDC definitions for nosocomial infections, 1988. Am J Infect Control. 1988;16:128-40. http://dx.doi.org/10.1016/01966553(88)90053-3

13. Rivers E, Nguyen B, Havstad S, Ressler J, Muzzin A, Knoblich B, et al. Early goal-directed therapy in the treatment of severe sepsis and septic shock. N Engl J Med. 2001;345:1368-77.

14. Kortgen A, Niederprum P, Bauer $\mathbf{M}$. Implementation of an evidence-based "standard operating procedure" and outcome in septic shock. Crit Care Med. 2006;34:943-9. http://dx.doi.org/10.1097/01.CCM.0000206112.32673.D4

15. Shapiro NI, Howell MD, Talmor D, Lahey D, Ngo L, Buras $\mathrm{J}$, et al. Implementation and outcomes of the Multiple Urgent Sepsis Therapies (MUST) protocol. Crit Care Med. 2006;34:1025-32. http://dx.doi.org/10.1097/01. CCM.0000206104.18647.A8

16. Micek ST, Roubinian N, Heuring T, Bode M, Williams $\mathrm{J}$, Harrison C, et al. Before-after study of a standardized hospital order set for the management of septic shock. Crit Care Med. 2006;34:2707-13. http://dx.doi.org/10.1097/01. CCM.0000241151.25426.D7

17. El Solh AA, Akinnusi ME, Alsawalha LN, Pineda LA. Outcome of septic shock in older adults after implementation of the sepsis "bundle". J Am Geriatr Soc. 2008;56:272-8. http://dx.doi.org/10.1111/j.1532-5415.2007.01529.x

18. Mikkelsen ME, Gaieski DF, Goyal M, Miltiades AN, Munson JC, Pines JM, et al. Factors associated with nonadherence to early goal-directed therapy in the ED. Chest. 2010;138:551-8. http://dx.doi.org/10.1378/chest.09-2210

19. Marshall JC, Dellinger RP, Levy M. The Surviving Sepsis Campaign:A history and a perspective. Surg Infect (Larchmt). 2010;11:275-81. http://dx.doi.org/10.1089/sur.2010.024.

20. Levy MM, Dellinger RP, Townsend SR, Linde-Zwirble WT, Marshall JC, Bion J, et al. The Surviving Sepsis Campaign: Results of an international guideline-based performance improvement program targeting severe sepsis. 
Crit Care Med. 2010;38:367-74. http://dx.doi.org/10.1097/ CCM.0b013e3181cb0cdc

21. Teles JM, Silva E, Westphal G, Filho RC, Machado FR. Surviving sepsis campaign in Brazil. Shock. 2008;30:47-52. http://dx.doi.org/10.1097/SHK.0b013e318181a128
22. Cardoso T, Carneiro AH, Ribeiro O, Teixeira-Pinto A, Costa-Pereira A. Reducing mortality in severe sepsis with the implementation of a core 6-hour bundle: Results from the Portuguese community-acquired sepsis study (SACiUCI study). Crit Care. 2010;14:R83. http://dx.doi.org/10.1186/ cc9008 